\title{
Joubert syndrome: accuracy of neuro-radiological findings
}

\author{
Sattam S. Lingawi ${ }^{1}$, Nahla A. Arab ${ }^{2}$, Yasser Ragab Fahmy ${ }^{3}$ \\ ${ }^{1}$ Department of Radiology, the University Hospital, King Abdulaziz University, Jeddah, Saudi Arabia \\ Departments of ${ }^{2}$ Pediatrics and ${ }^{3}$ Radiology, Dr. Erfan and Bagedo Hospital, Jeddah, Saudi Arabia
}

\begin{abstract}
To evaluate the accuracy of neuroimaging in establishing the diagnosis of Joubert syndrome. Computed tomography (CT) and magnetic resonance imaging (MRI) were performed in seven infants/children with the clinical diagnosis of Joubert syndrome. The clinical diagnoses were based on characteristic clinical features. The CT scans were performed on a single detector helical CT scans and the MRI were performed on 1-T and 1.5-T MRI systems. All studies were evaluated for the presence of cerebellar vermian aplasia/hypoplasia, cerebellar hypoplasia, horizontal alignment of the superior cerebellar peduncle, "molar tooth" configuration of the brainstem and "bat wing" appearance of the fourth ventricle. Other neuroimaging abnormalities such as hydrocephalus, dysgenesis of the corpus callosum were also reported. Cerebellar vermian hypoplasia with a midline cleft, horizontal alignment of the superior cerebellar peduncle, "molar tooth" configuration of the brainstem and dilated fourth ventricle with "bat wing" appearance in all patients. Cerebellar hemisphere hypoplasia was seen only in three cases. Brainstem molar tooth sign, fourth ventricular bat wing sign and the horizontal orientation of the superior cerebellar peduncles are characteristic findings Joubert syndrome. ( $\mathrm{J}$ Pediatr Neurol 2004; 2(1): 33-37).
\end{abstract}

Key words: Joubert syndrome, molar tooth sign, vermian hypoplasia.

Correspondence: Sattam S. Lingawi, M.D., FRCPC

P.O. Box 54403 Jeddah 21514,

Saudi Arabia.

E-mail: tersam2@yahoo.com

Tel: 9662-6408163

Received: May 10, 2003.

Revised: December 07, 2003.

Accepted: December 08, 2003.

\section{Introduction}

Joubert et al. (1) first reported Joubert syndrome (JS) in 1969, in four children having frequent attacks of alternate apnea and hyperpnoea, associated with hypotonia, ataxia, abnormal eye movements and psychomotor delay. Genetic defects in chromosome $9 q$ and WNT1 gene that is usually expressed in the developing cerebellum are believed to be linked to the occurrence of this syndrome $(2,3)$. However, the genetic examination cannot be used for the differential diagnosis of this syndrome, yet. In contrast, magnetic resonance imaging (MRI) plays a corner stone in establishing the diagnosis of Joubert syndrome. It usually reveals cerebellar hemispheres and/or vermian hypoplasia and a horizontally oriented superior cerebellar peduncles. Since the clinical symptoms of JS are highly variable, an accurate diagnostic tool is required. In this article we review and discuss the diagnostic accuracy of neuroimaging findings in cases of JS and present our experience with seven cases of this entity.

\section{Materials and Methods}

Computed tomography (CT) and MRI were performed in seven infants/children with the clinical suspicion of JS. The cases were referred to CT/MRI from the pediatric services of the contributing hospitals between the period of January 2000 and July 2003. All seven cases had both CT and MRI. The clinical diagnoses of JS were suspected on the basis of the physical findings including; coarse facies, low set ears, depressed nasal bridge, hypertelorism, bilateral polydactaly and irregular breathing pattern with alternating attacks of apnea and tachypnea. All seven patients were products of full term uneventful pregnancies with normal vaginal deliveries. Antenatal ultrasonography studies were not available. Due to service unavailability genetic analysis of the study population were not performed. The CT scans were performed on a single detector helical CT scans. All patients had a non-enhanced, non-helical axial 
Table 1. Clinical findings of the patients

\begin{tabular}{lllllll}
\hline \hline $\begin{array}{l}\text { Case } \\
\text { no }\end{array}$ & Age/sex & $\begin{array}{l}\text { Coarse } \\
\text { facies }\end{array}$ & $\begin{array}{l}\text { Low } \\
\text { set } \\
\text { ears }\end{array}$ & $\begin{array}{l}\text { Depressed } \\
\text { nasal } \\
\text { bridge }\end{array}$ & $\begin{array}{l}\text { Hypertelorism } \\
\text { Polydactyly }\end{array}$ & $\begin{array}{l}\text { Irregular } \\
\text { breathing }\end{array}$ \\
\hline 1 & 2 weeks/Female & + & + & + & + & + \\
2 & 3 days/Female & + & + & + & + & - \\
3 & 6 months/Male & + & + & + & - & - \\
4 & 2 months/Female & + & + & + & - & - \\
5 & 4 years/Male & + & + & - & - & - \\
6 & 5 months/Male & + & + & + & - & - \\
7 & 4 days/Male & + & + & - & + \\
\hline
\end{tabular}

CT scans of the brain at $5 \mathrm{~mm}$ slice thickness.

MRI were performed on a 1-T and 1.5-T MR systems. The imaging protocol included sagittal T1 spin echo, axial proton density, axial and coronal T2 fast spin echo and axial fluid attenuated inversion recovery (FLAIR). No contrast-enhanced images were obtained in any of the cases.

The first and third authors reported all cases prospectively. The images were reviewed again in a consensus fashion at the time of this study. All studies were evaluated for the presence of posterior fossa abnormalities including; cerebellar vermian aplasia/hypoplasia, cerebellar hypoplasia, horizontal alignment of the superior cerebellar peduncle, "molar tooth" configuration of the brainstem and "bat wing" appearance of the fourth ventricle. Other neuroimaging abnormalities such as hydrocephalus, dysgenesis of the corpus callosum were also sought.

\section{Results}

Clinically all patients had coarse facies and low set ears. Five patients had depressed nasal bridge. Three patients had hypertelorism and only one patient had polydactyly (left extra little finger). Irregular breathing pattern with alternating attacks of apnea and tachypnea were present in six patients. (Table 1). The parents were consanguineous in only three cases (cases 1-3).

Neuroimaging revealed vermian hypoplasia with a midline cleft, horizontal alignment of the superior cerebellar peduncle, "molar tooth" configuration of the brainstem and dilated fourth ventricle with "bat wing" appearance in all patients (Figures 1-3). Hypoplasia of Cerebellar hemisphere was seen in only three cases. None of the cases had complete cerebellar or vermian aplasia. No hydrocephalus, dysgenesis of the corpus callosum or other posterior fossa abnormalities were detected.

\section{Discussion}

Phenotype

$\mathrm{JS}$ is a rare autosomal recessive disorder. The phenotypic features of JS are highly variable making it difficult to establish the exact clinical diagnostic features of the syndrome (3). Among the commonly reported clinical features are hypotonia, coarse facies, depressed nasal bridge, low set ears hypertelorism with abnormal eye movements, abnormal breathing patterns with attacks of apnea and tachypnea and developmental delay. Other associated features include polydactyly (8\%), coloboma $(2 \%)$, renal cyst $(2 \%)$ and soft tissue tumors of the tongue (2). All these features were present in our series (4).

The genetic and biochemical basis of this syndrome remain vastly unspecified. Genetic mapping and chromosomal analysis were not performed in our study due to their local unavailability.

\section{Classification}

Children with JS can be classified into two distinct groups. In the severe form of the disease, patients present with coarse facies, neonatal seizures and severe respiratory fluctuating pattern. Those patients usually die at young age due to recurrent chest infection and worsening respiratory compromise. We believe that six out of the seven patients in this study belong to this group. The second group represents the milder form of the disease. These patients usually survive and show variable degrees of mental and cognitive impairments. Respiratory irregularities are usually minimal or absent in this group (6). In our renes only one patient (case 5) belongs to this group.

\section{Neuroimaging}

MRI plays a corner stone in establishing the diagnosis of JS. It usually reveals cerebellar hemispheres and/or vermian hypoplasia and a horizontally oriented superior cerebellar peduncles. Subsequently, the fourth ventricle is usually moderately dilated taking the appearance of a "bat wing" or an open "umbrella" on axial CT/MRI. The axial CT/MRI also reveal the brainstem to be of an unusually deformed configuration simulating a 


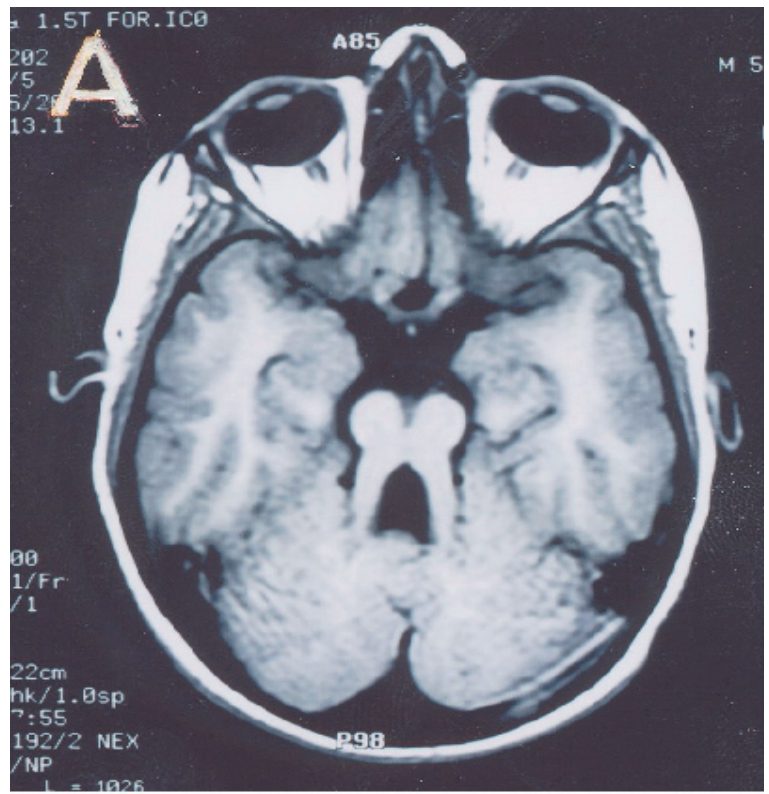

Figure 1a. Axial T1 spin echo MRI illustrating the classic molar tooth appearance of the brainstem in case 1 .

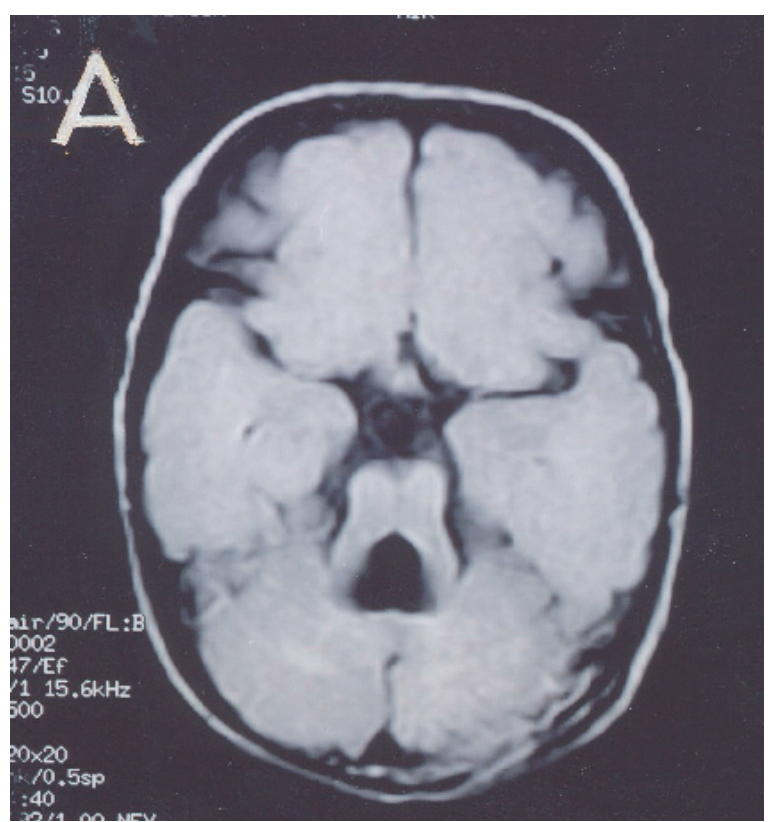

Figure 2a. In case 4 axial T1 spin echo MRI at the level fourth ventricle revealing the ventricle to be mildly dilated with an unusual configuration simulating the appearance of an open umbrella.

"molar tooth". The later two signs are reported to be consistent and reliable imaging findings to distinguish JS from other posterior fossa abnormalities $(7,8)$.

These findings were present in all seven patients in this study. They are best demonstrated on axial imaging plane and could be easily missed on sagittal and coronal images. In comparison horizontal orientation of the superior cerebellar peduncle (seen in all of our patients) are best visualized on sagittal para-median images.

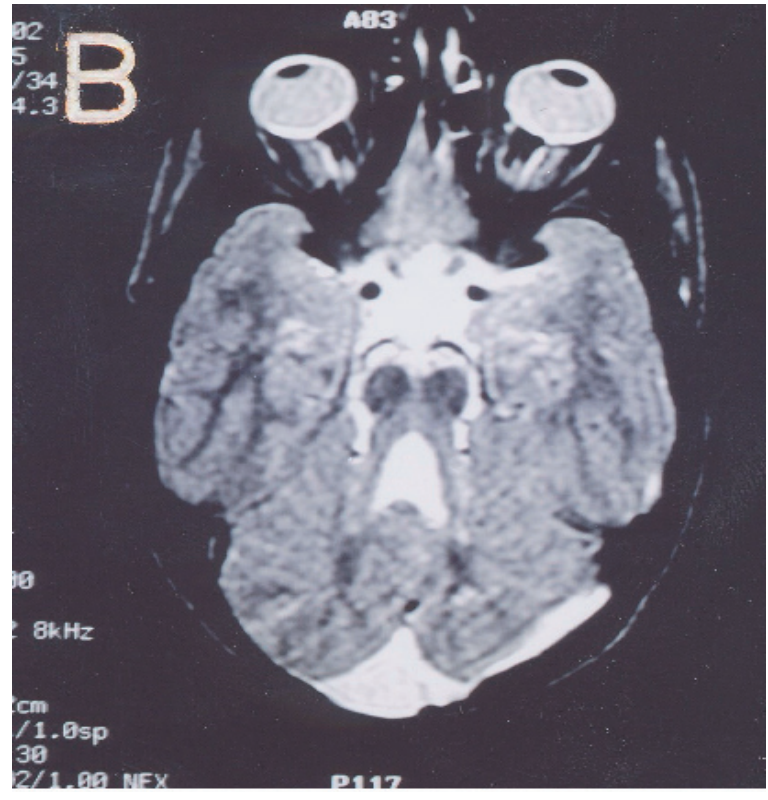

Figure 1b. Axial T2 spin echo MRI illustrating the classic molar tooth appearance of the brainstem in case 1 .

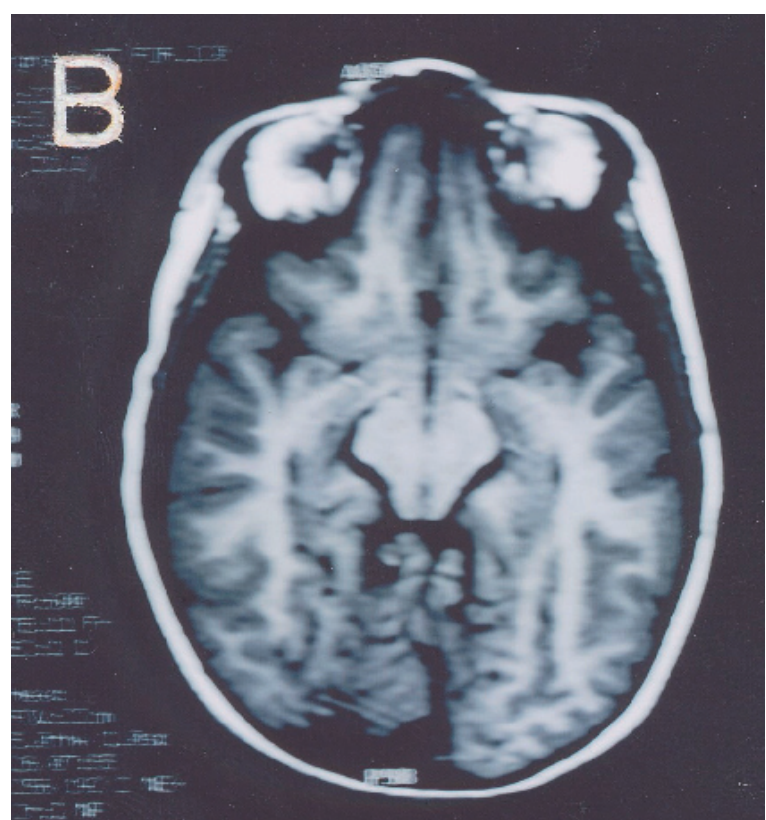

Figure 2b. In case 4 axial T1 spin echo MRI at the level of lower midbrain revealing hypoplasia and abnormal configuration of the vermis with a midline cleft.

Other MRI findings including hydrocephalus, corpus callosum agenesis, posterior fossa cyst and Dandy-Walker malformation; were not present in our study population. These are infrequently reported in documented cases of JS (9).

The respiratory irregularities are believed to be secondary to the brainstem and cerebellar abnormalities; however, the developmental delay and cognitive dysfunction cannot be solely explained by the basis of hindbrain abnormality and is probably due to other cerebral hemispheres 


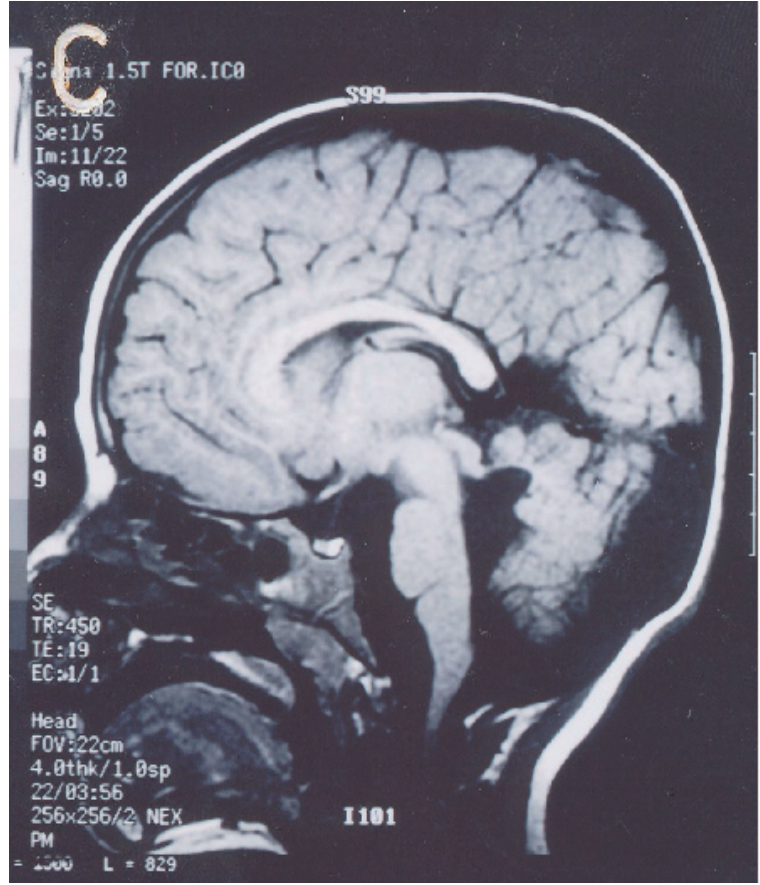

Figure 2c. In case 4, midline sagittal $\mathrm{T} 1$ spin echo MRI revealing mild dilatation and abnormal configuration of the fourth ventricle with upward deviation and horizontal direction of the superior cerebellar peduncles. Mildly enlarged cisterna magna is also noted.

dysfunction $(10,11)$.

\section{Conclusion}

In conclusion, we believe that the brainstem molar tooth sign, the fourth ventricular bat wing sign and the horizontal orientation of the superior cerebellar peduncles are characteristic posterior fossa neuroimaging findings that are present in all cases of JS. Such findings when present together makes the possibility of alternative diagnoses rather unlikely.

\section{Editorial note}

Since this paper was accepted for publication, two papers $(13,14)$ have argued that the molar tooth sign on imaging (MTI) is not specific for JS. At present it is a matter of argument whether these various conditions with MTI with one or more retinal, renal, digital or other anomaly are subtypes of JS or whether they are separate entities. From the point of view of genetic analysis, the latter approach-splitting rather than lumping-may be preferred.

\section{References}

1. Chance PF, Cavalier L, Satran D, Pellegrino JE, Koenig M, Dobyns WB. Clinical nosologic and genetic aspects of Joubert and related syndromes. J

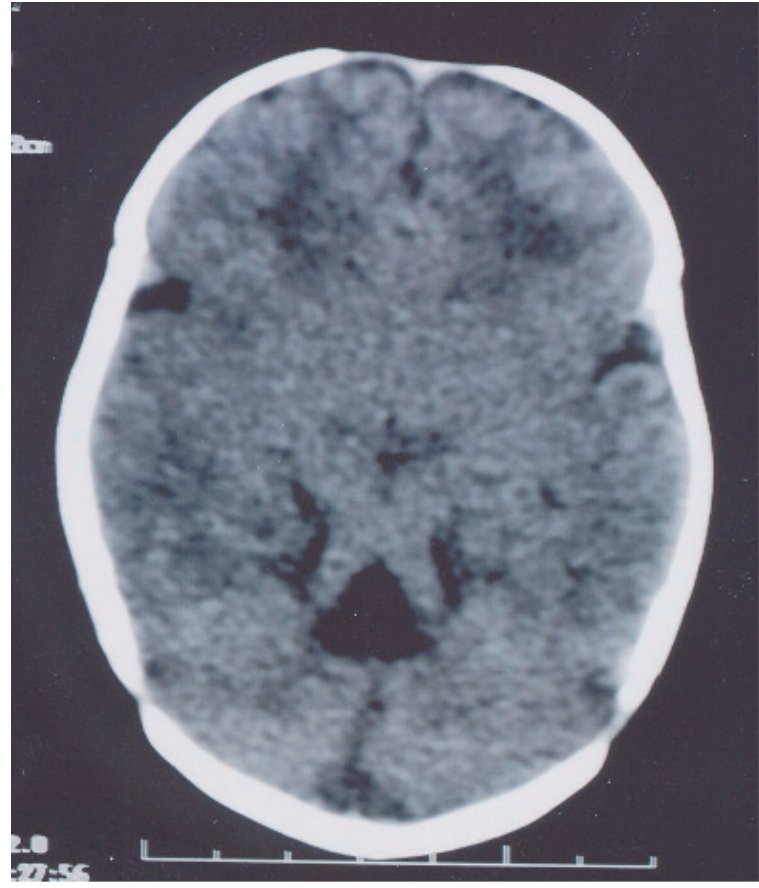

Figure 3. In case 6, axial non-enhanced CT scan of the brain at the level of the pons revealing the classic molar tooth appearance of the brainstem.

Child Neurol 1999; 14: 660-666.

2. Joubert M, Eisenring JJ, Robb JP, Andermann F. Familial agenesis of the cerebellar vermis. A syndrome of episodic hyperpnea, abnormal eye movements, ataxia and retardation. Neurology 1969 ; 19: $813-825$.

3. Saar K, Al-Gazali L, Sztriha L, et al. Homozygosity mapping in families with Joubert syndrome identifies a locus on chromosome $9 \mathrm{q} 34.3$ and evidence for genetic heterogeneity. Am J Hum Genet 1999; 65: 1666-1671.

4. Barreirinho MS, Teixeira J, Moreira NC, Bastos S, Goncalvez S, Barbot MC. Joubert's syndrome: report of 12 cases. Rev Neurol 2001; 32: $812-817$ (in Spanish).

5. Aslan H, Ülker V, Gülcan EM, et al. Prenatal diagnosis of Joubert syndrome: a case report. Prenat Diagn 2002; 22: 13-16.

6. Steinlin M, Schmid M, Landau K, Boltshauser E. Follow-up in children with Joubert syndrome. Neuropediatrics 1997; 28: 204-211.

7. Maria BL, Bozorgmanesh A, Kimmel KN, Theriaque D, Quisling RG. Quantitative assessment of brainstem development in Joubert syndrome and Dandy-Walker syndrome. J Child Neurol 2001; 16: 751-758.

8. Yachnis AT, Rorke LB. Cerebellar and brainstem development: an overview in relation to Joubert syndrome. J Child Neurol 1999; 14: 570-573.

9. Zamponi N, Rossi B, Messori A, Polonara G, Regnicolo L, Cardinali C. Joubert syndrome with associated corpus callosum agenesis. Eur J Paediatr Neurol 2002; 6: 63-66.

10. Maria BL, Boltshauser E, Palmer SC, Tran TX. 
Clinical features and revised diagnostic criteria in Joubert syndrome. J Child Neurol 1999; 14: 583590.

11. Saito $\mathrm{Y}$, Ito $\mathrm{M}$, Ozawa $\mathrm{Y}$, et al. Changes of neurotransmitters in the brainstem of patients with respiratory-pattern disorders during childhood. Neuropediatrics 1999; 30: 133-140.

12. Graber D, Antignac C, Deschenes G, et al. Cerebellar vermis hypoplasia with extracerebral involvement (retina, kidney, liver) difficult to classify syndromes.
Arch Pediatr 2001; 8: 186-190 (in French).

13. Valente EM, Salpietro DC, Brancati F, et al Description, nomenclature, and mapping of a novel cerebello-renal syndrome with the molar tooth malformation. Am J Hum Genet 2003; 73: 663-70.

14. Parisi MA, Dobyns WB. Human malformations of the midbrain and hindbrain: review and proposed classification scheme. Mol Genet Metab 2003; 80: 36-53. 